\title{
Efeitos da relação metionina + cistina:lisina sobre os desempenhos produtivo e econômico e a qualidade interna e externa dos ovos antes e após 28 dias de armazenamento ${ }^{1}$
}

\author{
José Jordão Filho², José Humberto Vilar da Silva ${ }^{3}$, Edson Lindolfo da Silva ${ }^{4}$, Daniel de \\ Magalhães Araujo5, Marcelo Luís Gomes Ribeiro ${ }^{2,6}$, Matheus Ramalho de Lima ${ }^{7}$
}

\footnotetext{
${ }^{1}$ Parte da dissertação de Mestrado do primeiro autor apresentada ao PPGZ/CCA/UFPB, Areia - PB

2 Doutorado Integrado em Zootecnia, PDIZ/CCA/UFPB, Areia - PB. Bolsista CAPES.

${ }^{3} \mathrm{DAP} / \mathrm{CFT} / \mathrm{UFPB}$, Bananeiras - PB e PPGZ - CCA/UFPB, Areia, PB. Bolsista PQ - CNPq.

${ }^{4}$ Doutorando em Zootecnia, UFLA - Lavras, MG.

5 Mestre em Zootecnia, PPGZ/CCA/UFPB, Areia - PB.

${ }^{6}$ DAP/CFT/UFPB, Bananeiras - PB.

7 Curso de Licenciatura em Ciências Agrárias, CFT/UFPB, Bananeiras - PB.
}

RESUMO - Avaliou-se o efeito de diferentes relações metionina + cistina (Met+Cis):lisina (Lis) total sobre o desempenho e a qualidade de ovos para poedeiras semipesadas. Foram utilizadas 120 aves da linhagem Lohmann Brown com 44 semanas de idade, alimentadas com rações isonutritivas (17\% PB, 2.774 kcal EMAn, 4,28\% de Ca e 0,375\% Pd) e distribuídas em um delineamento inteiramente casualizado, com cinco repetições e quatro tratamentos, constituídos da seguinte forma: $\mathrm{T}_{1}=$ relação 0,76 ou $0,70 \%$ de Met + Cis e $0,92 \%$ de Lis total; $\mathrm{T}_{2}=$ relação 0,83 ou $0,71 \%$ de Met + Cis e $0,85 \%$ de Lis total; $\mathrm{T}_{3}=$ relação 0,85 ou $0,64 \%$ Met + Cis e $0,75 \%$ de Lis total; $\mathrm{T}_{4}=$ relação 0,86 ou $0,70 \%$ de Met+Cis e $0,81 \%$ de Lis total. As variáveis de desempenho e o resultado da análise econômica foram estudadas no período de 44 a 56 semanas de idade das poedeiras. Ao término do experimento, dez ovos por tratamento foram coletados e armazenados por 28 dias para avaliação da qualidade interna e externa dos ovos antes e após a armazenagem. À exceção da massa de ovos, os tratamentos não afetaram o consumo de ração, a produção, o peso, a conversão por massa e por dúzia de ovos e a gravidade específica da casca. A relação Met+Cis: Lis de 0,76 ou a estimativa de $0,70 \%$ de Met+Cis total e $0,92 \%$ de Lis total podem ser recomendadas para alimentação de poedeiras semipesadas. Concluiu-se que o armazenamento afeta a qualidade interna dos ovos.

Palavras-chave: aminoácidos, análise econômica, desempenho, qualidade do ovo

\section{Effects of methionine + cystine:lysine ratio on the productive and economic performance and internal and external egg quality, before and 28 days after storage}

\begin{abstract}
The effect of different methionine + cystine (Met+Cis):total lysine (Lis) ratios on performance and egg quality for semi-heavily laying hens was evaluated. One hundred and twenty Lohmann Brown hens with 44 weeks old were allotted to a completely randomized design with with five replicates and four treatments, as follows: $\mathrm{T}_{1}=0.76 \mathrm{Met}+\mathrm{Cys}$ :total Lys ratio or $0.70 \%$ of Met+Cys and $0.92 \%$ of total Lys; $\mathrm{T}_{2}=0.83$ Met+Cys: total Lis ratio or $0.71 \%$ of Met + Cys and $0.85 \%$ of total Lys; $\mathrm{T}_{3}=0.85$ Met+Cys: total Lys ratio or $0.64 \%$ of Met + Cys and $0.75 \%$ of total Lys; $\mathrm{T}_{4}=0.86$ Met + Cys: total Lys ratio or $0.70 \%$ of Met+Cys and $0.81 \%$ of total Lys. The performance variables and economical analysis were evaluated from 44 to 56 weeks old. At the end of trial, ten eggs per treatment were collected ad stored during 28 days for evaluation of internal/external egg quality before and after storage. With the exception of egg mass, no treatment effect on feed intake, egg production, egg weight and egg:mass ratio and egg:dozen ratio and egg shell specific quality was observed. The Met + Cys:Lys ratio of 0.76 or the estimate of $0.70 \%$ of total Met+Cys and $0.92 \%$ of total Lys can be recommended for the feeding of semiheavily laying hens. It was concluded that the storage affect internal egg quality.
\end{abstract}

Key Words: amino acids, economic analysis, egg quality, performance

\section{Introdução}

A formulação de dietas para poedeiras semipesadas está, geralmente, condicionada aos níveis nutricionais preconizados nas tabelas e nos manuais de criação, a partir de informações obtidas de forma isolada e, portanto, podem não ser tão eficientes na otimização do desempenho das aves quanto àquelas estimadas com base na relação dos aminoácidos essenciais (Silva et al., 2005). Além disso, alguns questionamentos recaem sobre os níveis de 
metionina+cistina e de lisina recomendados pelo NRC (1994), considerados inadequados para poedeiras (Yadalan et al., 2000) por onerar o preço da ração.

O ponto crítico da produção avícola é o gasto com alimentação, especialmente com as especificações nutricionais de proteína e energia, que contribuem com a maior parte do preço da ração. Assim, diferentes relações aminoácidos essenciais:lisina vêm sendo pesquisadas visando atender melhor às exigências nutricionais das aves (Silva et al., 2002, Silva et al., 2004ab). Poucos trabalhos têm associado o desempenho das aves ao custo de alimentação e à rentabilidade econômica da produção de ovos, em virtude da falta de métodos adequados para essa análise.

A qualidade interna e externa dos ovos não tem merecido a devida atenção nos estudos nutricionais realizados com aves de postura, considerando, especialmente, os efeitos das especificações dos aminoácidos das dietas sobre o ovo como alimento humano. A influência dos aminoácidos sobre a qualidade do ovo armazenado em geladeira doméstica também tem sido pouco estudada.

A altura do albúmen do ovo tem sido adotada como critério de avaliação da qualidade interna dos ovos, sendo medida pela unidade Haugh. Entretanto, Eisen et al. (1962) criticaram o uso da unidade Haugh por ser medida em relação ao peso do ovo, visto que esta equação não considera o tamanho e a proporção dos componentes do ovo das diferentes linhagens e da idade das poedeiras. Por outro lado, o $\mathrm{pH}$ deve ser uma medida mais adequada para descrever a qualidade do albúmen após longo período de estocagem. Entretanto, as diferenças no $\mathrm{pH}$ não são associadas à qualidade do ovo fresco (Silversides \& Villeneuve, 1994), possivelmente porque o ovo não proporciona condições para multiplicação de microrganismos patogênicos. O uso da equação de Haugh foi defendido para ovos frescos, mas não para ovos estocados (Kidwell et al., 1964), provavelmente em virtude da redução da altura do albúmen com o período de armazenagem. Silversides et al. (1993) sugeriram que a medida da altura do albúmen é suficiente para avaliar a qualidade interna de ovos frescos, à exceção daqueles de poedeiras em diferentes idades, para as quais não deve ser usada.

O aumento no desempenho de poedeiras com a suplementação de aminoácidos têm sido avaliado em muitos trabalhos realizados nos últimos anos e merece atenção quanto à influência dos aminoácidos sobre a qualidade interna dos ovos em condições de estocagem. Shafer et al. (1996) observaram aumento no peso e na quantidade de sólidos totais do albúmen e da gema dos ovos quando a suplementação de metionina foi elevada de 326 para $512 \mathrm{mg} /$ ave/dia. Similarmente, Shafer et al. (1998) verificaram elevado nível de proteína dos componentes do ovo com a suplementação de 507 em comparação a 413 mg de metionina/ ave/dia. Em estudos de exigência de lisina, Prochaska et al. (1996) encontraram maior peso do albúmen e da gema com 828 mg e maior proteína do albúmen com 1.062 mg em relação ao nível de $628 \mathrm{mg} / \mathrm{ave} / \mathrm{dia}$ de lisina. Portanto, novos estudos relacionando a qualidade interna do ovo à suplementação de aminoácidos podem ser de grande interesse na indústria de ovo líquido. Assim, realizou- se este estudo com o objetivo de avaliar, nutricional e economicamente, diferentes relações metionina + cistina:lisina recomendadas para poedeiras semipesadas e seus efeitos sobre a qualidade interna e externa dos ovos após 28 dias de estocagem.

\section{Material e Métodos}

O experimento foi conduzido no Núcleo de Pesquisa em Aves do Centro de Formação de Tecnólogos (CFT), Campus III, da Universidade Federal da Paraíba (UFPB), situado no município de Bananeiras, na microrregião do Brejo Paraibano. Foram utilizadas 120 poedeiras semipesadas da linhagem Lohmann Brown, com 44 semanas de idade e peso vivo de $1,8 \pm 0,057 \mathrm{~kg}$. A seleção das aves foi realizada com base no peso vivo e na produção de ovos, avaliados durante 15 dias antes do período experimental. As aves foram submetidas a um programa de iluminação de 17 horas/ dia (luz natural + artificial).

A parcela experimental, constituída de seis aves, ocupava três compartimentos $(25 \times 45 \times 40 \mathrm{~cm}$, com duas aves cada um) de gaiolas convencionais, em um galpão experimental com cobertura de telhas de barro em duas águas, orientação Leste-Oeste, pé-direito de 1,8 $\mathrm{m}$ de altura, duas fileiras de gaiolas sobrepostas e um corredor de $1 \mathrm{~m}$ de largura entre as fileiras de gaiolas. O experimento foi realizado no período de 44 a 56 semanas de idade das aves, sendo dividido em quatro fases experimentais de 21 dias.

Foi adotado o delineamento experimental inteiramente casualizado, com quatro tratamentos de cinco repetições, caracterizados pelas seguintes relações metionina + cistina: lisina (met+cis:lis):

$\mathrm{T}_{1}=$ relação met + cis:lis de 0,76 , correspondente a $0,70 \%$ met + cis e $0,92 \%$ lis total (Jordão Filho et al., 2006ab);

$\mathrm{T} 2$ = relação met+cis:lis de 0,83 , correspondente a $0,71 \%$ met + cis e $0,85 \%$ lis total (Interaves, 1999 ).

$\mathrm{T} 3=$ relação met + cis:lis de 0,85 , correspondente a $0,64 \%$ met + cis e $0,75 \%$ lis total (NRC, 1994 );

$\mathrm{T} 4=$ relação met + cis:lis de 0,86 , correspondente a $0,70 \%$ met + cis e $0,81 \%$ lis total (Rostagno et al., 2000). 
Os níveis dos aminoácidos das rações foram alterados pela manipulação dos níveis de DL-metionina, L-lisina $\mathrm{HCl}$ e amido de milho das dietas. A metionina e lisina nas fontes comerciais DL-metionina (99\% de atividade de metionina) e L-lisina $\mathrm{HCl}(78,4 \%$ de atividade de lisina), respectivamente, foram consideradas como $100 \%$ digestíveis. As rações estudadas, isoprotéicas e isocalóricas (Tabela 1), foram formuladas para atender aos requerimentos nutricionais de poedeiras semipesadas, segundo Rostagno et al. (2000), exceto as relações met + cis:lis total de 0,$76 ; 0,83$ e 0,85 .

As composições protéica e energética dos ingredientes são apresentadas na Tabela 2, enquanto os valores totais

Tabela 1 - Composição das rações experimentais ${ }^{1}$

Table 1 - Ingredient and chemical compositions of the experimental diets

\begin{tabular}{|c|c|c|c|c|}
\hline \multirow[t]{2}{*}{$\begin{array}{l}\text { Item } \\
\text { Item }\end{array}$} & \multicolumn{4}{|c|}{$\begin{array}{c}\text { Relação met+cis:lis total }(\%) \\
\text { Total met+cys:cys ratio }\end{array}$} \\
\hline & 0,76 & 0,83 & 0,85 & 0,86 \\
\hline Milho (Corn) & 61,860 & 61,860 & 61,860 & 61,860 \\
\hline Farelo de soja (Soybean meal) & 19,960 & 19,960 & 19,960 & 19,960 \\
\hline Glúten de milho (Corn gluten) & 4,200 & 4,200 & 4,200 & 4,200 \\
\hline Calcário (Limestone) & 9,940 & 9,940 & 9,940 & 9,940 \\
\hline Fosfato bicálcico (Dicalcium phosphate) & 1,510 & 1,510 & 1,510 & 1,510 \\
\hline Amido (Starch) & 0,825 & 0,935 & 1,011 & 0,923 \\
\hline DL-metionina $(99 \%)$ (DL-methionine) & 0,118 & 0,125 & 0,059 & 0,112 \\
\hline L-lisina $\mathrm{HCl}(78,4 \%)$ (L-lysine $\mathrm{HCl})$ & 0,187 & 0,125 & 0,000 & 0,074 \\
\hline Cloreto de colina $(70 \%)$ (Choline chloride) & 0,200 & 0,200 & 0,200 & 0,200 \\
\hline Óleo vegetal (Vegetable oil) & 0,450 & 0,450 & 0,450 & 0,450 \\
\hline Sal comum (Salt) & 0,550 & 0,550 & 0,550 & 0,550 \\
\hline Premix mineral ${ }^{2}$ (Mineral mix) & 0,200 & 0,200 & 0,200 & 0,200 \\
\hline Premix vitamínico ${ }^{3}$ (Vitamin mix) & 0,050 & 0,050 & 0,050 & 0,050 \\
\hline Areia lavada (Washed sand) & 0,010 & 0,010 & 0,010 & 0,010 \\
\hline Total & 100,000 & 100,000 & 100,000 & 100,000 \\
\hline
\end{tabular}

Composição química

Chemical composition

\begin{tabular}{|c|c|c|c|c|}
\hline Proteína bruta (\%) (Crude protein) & 17,000 & 17,000 & 17,000 & 17,000 \\
\hline EMAn (kcal/kg) (AMEn) & 2.774 & 2.774 & 2.774 & 2.774 \\
\hline Gordura (\%) (Fat) & 2,990 & 2,990 & 2,990 & 2,990 \\
\hline Cálcio (\%) (Calcium) & 4,280 & 4,280 & 4,280 & 4,280 \\
\hline Fósforo disponível (\%) (Available phosphorus) & 0,372 & 0,372 & 0,372 & 0,372 \\
\hline Metionina+cistina $(\%) \mathrm{T}^{4}($ Met + Cys $)$ & 0,700 & 0,710 & 0,643 & 0,700 \\
\hline Metionina+cistina $(\%) \mathrm{D}^{4}($ Met + Cys $)$ & 0,640 & 0,640 & 0,580 & 0,630 \\
\hline Metionina (\%) $\mathrm{T}$ (Methionine) & 0,310 & 0,380 & 0,330 & 0,385 \\
\hline Metionina (\%) D (Methionine) & 0,290 & 0,340 & 0,297 & 0,346 \\
\hline Lisina $(\%) \mathrm{T}$ (Lysine) & 0,920 & 0,850 & 0,750 & 0,811 \\
\hline Lisina (\%) D (Lysine) & 0,840 & 0,770 & 0,670 & 0,718 \\
\hline Arginina $(\%) \mathrm{T}$ (Arginine) & 0,912 & 0,912 & 0,912 & 0,912 \\
\hline Arginina (\%) D (Arginine) & 0,852 & 0,852 & 0,852 & 0,852 \\
\hline Isoleucina (\%) $\mathrm{T}$ (Isoleucine) & 0,601 & 0,601 & 0,601 & 0,601 \\
\hline Treonina (\%) T (Threonine) & 0,647 & 0,647 & 0,647 & 0,647 \\
\hline Treonina (\%) D (Threonine) & 0,483 & 0,483 & 0,483 & 0,483 \\
\hline Triptofano (\%) $\mathrm{T}$ (Tryptophan) & 0,179 & 0,179 & 0,179 & 0,179 \\
\hline Triptofano (\%) D (Tryptophan) & 0,150 & 0,150 & 0,150 & 0,150 \\
\hline Leucina (\%) $\mathrm{T}$ (Leucine) & 1,348 & 1,348 & 1,348 & 1,348 \\
\hline Leucina (\%) D (Leucine) & 1,240 & 1,240 & 1,240 & 1,240 \\
\hline Valina (\%) $\mathrm{T}$ (Valine) & 0,673 & 0,673 & 0,673 & 0,673 \\
\hline Valina (\%) D (Valine) & 0,594 & 0,594 & 0,594 & 0,594 \\
\hline Sódio (\%) (Sodium) & 0,251 & 0,251 & 0,251 & 0,251 \\
\hline Cloro (\%) (Chlorine) & 0,397 & 0,385 & 0,361 & 0,375 \\
\hline Potássio (\%) (Potassium) & 0,563 & 0,563 & 0,563 & 0,563 \\
\hline Balanço eletrolítico (Electrolyte balance) & 141,182 & 144,566 & 151,336 & 147,387 \\
\hline Preço (R\$/kg) (Price) & 0,6350 & 0,6336 & 0,6186 & 0,6268 \\
\hline
\end{tabular}


e digestíveis de lisina e de metionina+cistina, do milho, do farelo de soja e do glúten de milho, tomados como base para a estimativa, na Tabela 3 .

A água foi fornecida à vontade, mas a oferta de ração farelada foi equalizada em $110 \mathrm{~g} / \mathrm{ave} / \mathrm{dia}$, ou consumo estimado de $308 \mathrm{kcal} / \mathrm{ave} / \mathrm{dia}$, suficiente para atender às necessidades de mantença e produção segundo o modelo de predição preconizado por Rostagno et al. (2000), considerando $1,6 \mathrm{~kg}$ como peso vivo médio das aves, $1 \mathrm{~g}$ de ganho de peso diário e $55 \mathrm{~g}$ de massa de ovos produzida.

As variáveis de desempenho estudadas foram consumo de ração (g/ave/dia), produção de ovos (\%/ave/dia), peso (g) e massa de ovos (g/ave/dia), conversão alimentar por massa $(\mathrm{kg} / \mathrm{kg})$, conversão alimentar por dúzia de ovos $(\mathrm{kg} / \mathrm{dz})$ e gravidade específica $\left(\mathrm{g} / \mathrm{cm}^{3}\right)$.

O consumo de ração foi calculado como a diferença entre a quantidade de ração fornecida e as sobras. A produção de ovos foi obtida em porcentagem/ave/dia, enquanto o peso dos ovos foi o valor médio do peso dos ovos produzidos nos últimos cinco dias de cada fase experimental. A massa de ovos foi determinada pelo produto da porcentagem dos ovos produzidos e o peso médio dos ovos; a conversão alimentar por massa de ovos, pela relação

Tabela 2 - Composição dos ingredientes utilizados nas rações Table 2 - Ingredient composition of the diets

\begin{tabular}{|c|c|c|}
\hline $\begin{array}{l}\text { Ingrediente } \\
\text { Ingredient }\end{array}$ & $\begin{array}{c}\mathrm{PB}(\%) \\
C P\end{array}$ & $\begin{array}{c}\text { EMAn }(\mathrm{kcal} / \mathrm{kg}) \\
M E\end{array}$ \\
\hline Milho' $^{1}$ (Corn) & 8,57 & 3.371 \\
\hline Farelo de soja $^{1}$ (Soybean meal) & 45,54 & 2.266 \\
\hline Glúten de milho $(60 \%)^{1}$ (Corn gluten) & 59,85 & 3.775 \\
\hline Amido $^{1}$ (Starch) & 0,55 & 3.625 \\
\hline DL-metionina $(0,99 \%)^{2}$ (DL-methionine) & 58,70 & 3.680 \\
\hline L-lisina $\cdot \mathrm{HCl}(78,4 \%)^{2}$ (L-lysine $\left.\mathrm{HCl}\right)$ & 119,80 & 4.600 \\
\hline Óleo vegetal (Vegetable oil) & 0,00 & 8.790 \\
\hline
\end{tabular}

1 Segundo Rostagno et al. (2000) (According to Rostagno et al., 2000 tables).

2 Segundo NRC (1994) (According to NRC, 1994 tables).

Tabela 3 - Valores totais e digestíveis de lisina e de metionina+cistina dos alimentos 1

Table 3 - Digestible and total values of lysine and methionine+cystine of the feedstuffs

\begin{tabular}{|c|c|c|c|c|}
\hline \multirow[t]{2}{*}{$\begin{array}{l}\text { Alimento } \\
\text { Feedstuff }\end{array}$} & \multicolumn{2}{|c|}{$\begin{array}{l}\text { Lisina }(\%) \\
\text { Lysine }\end{array}$} & \multicolumn{2}{|c|}{$\begin{array}{c}\text { Metionina }+ \text { cistina }(\%) \\
\text { Methionine }+ \text { cystine }\end{array}$} \\
\hline & $\begin{array}{l}\text { Total } \\
\text { Total }\end{array}$ & $\begin{array}{c}\text { Digestível } \\
\text { Digestible }\end{array}$ & $\begin{array}{l}\text { Total } \\
\text { Total }\end{array}$ & $\begin{array}{l}\text { Digestível } \\
\text { Digestible }\end{array}$ \\
\hline $\begin{array}{l}\text { Milho } \\
\text { Corn }\end{array}$ & 0,25 & 0,203 & 0,37 & 0,325 \\
\hline $\begin{array}{l}\text { Farelo de soja } \\
\text { Soybean meal }\end{array}$ & 2,78 & 2,530 & 1,27 & 1,120 \\
\hline $\begin{array}{l}\text { Glúten de milho }(60 \%) \\
\text { Corn gluten }\end{array}$ & 1,00 & 0,900 & 2,50 & 2,300 \\
\hline
\end{tabular}

\footnotetext{
1 Valores constantes em Rostagno et al. (2000) (According to Rostagno et al.,
} 2000tables) entre o quilograma de ração consumido por quilograma de ovo produzido; e a conversão alimentar por dúzia de ovos, pela relação entre a quantidade de ração consumida $(\mathrm{kg})$ por dúzia de ovos produzida.

A gravidade específica foi avaliada pelo método da flutuação dos ovos em 15 soluções salinas diferentes quanto às densidades, que variaram em 0,0025 unidades, iniciando em 1,0625 até 1,100 . Foram utilizados baldes com capacidade de $50 \mathrm{~L}$, um densímetro de petróleo com escala de 1,050 a 1,100 , uma cesta de plástico (balde vasado), água e sal comum.

Dois ovos por parcela foram armazenados em geladeira doméstica durante 28 dias, a $5^{\circ} \mathrm{C}$, e foram usados nas análises da qualidade interna e externa dos ovos, sendo a primeira realizada com base nos conteúdos de clara e de gema e a segunda, considerando-se a resistência da casca, medida pelo peso e porcentagem de casca e pela gravidade específica $\left(\mathrm{g} / \mathrm{cm}^{3}\right)$, antes e depois do armazenamento. A porcentagem da casca foi calculada em relação ao peso do ovo e a gravidade específica foi determinada pela metodologia de flutuação dos ovos em solução salina, descrita anteriormente.

A qualidade interna dos ovos foi avaliada pelo potencial hidrogeniônico $(\mathrm{pH})$ e pelo potencial de óxido-redução (Eh), medidos por peagâmetro modelo Tec-2. O pH e o Eh são medidas indiretas da inibição ou da multiplicação dos microrganismos durante o período de estocagem de um alimento. Pelo pH, obtém-se a variação de concentração de íons hidrogênio no interior do ovo (gema e clara), enquanto, pelo Eh, estima-se a facilidade com que o substrato capta ou cede elétrons. Portanto, o potencial redox sugere a presença ou ausência de microrganismos aeróbicos e anaeróbicos no material.

A análise econômica foi realizada considerando os seguintes índices: renda média bruta (RMB), custo médio de arraçoamento (CMA), margem bruta média (MBM) e rentabilidade média do ovo (RMO). Este método foi originalmente desenvolvido para avaliar o resultado econômico da criação de frangos de corte (Guidoni, 1994), tendo sido feito adaptação inédita à análise econômica da produção de ovos (Silva et al., 2005). Assim, a RMB foi obtida multiplicando-se a produção de ovos durante o experimento, em número de caixas com 30 unidades, pelo preço de venda de $\mathrm{R} \$ 3,70$. O CMA foi calculado como o produto do consumo de ração em cada tratamento pelo preço da ração $(\mathrm{kg})$, enquanto a MBM representa a diferença entre a RMB e o CMA e a RMO indica a porcentagem da MBM em relação ao CMA. Para o desenvolvimento dos índices, foram tomados como referência os preços dos ingredientes, descritos na Tabela 4. 
As análises estatísticas foram realizadas utilizando-se o software SAEG - Sistema de Análise Estatística e Genética, versão 5.0, desenvolvido pela Universidade Federal de Viçosa - UFV (1993). Para escolha da melhor relação, foi adotado o teste Duncan $(\mathrm{P}<0,05)$.

\section{Resultados e Discussão}

O desempenho produtivo das aves, de acordo com as diferentes relações met+cis:lis, está descrito na Tabela 5 .

O consumo de ração não foi alterado pelos tratamentos $(\mathrm{P}>0,05)$, o que indica que as diferentes recomendações dos aminoácidos das dietas provavelmente não causaram efeito redutor do desbalanço aminoacídico sobre o consumo de ração das aves. Segundo Nunes (1998) e Silva et al. (2000abc), o desbalanço ocorre quando um aminoácido essencial é adicionado cerca de $2 \%$ acima das exigências nutricionais, pela desproporção na relação com os outros aminoácidos essenciais.

A produção, o peso e as conversões por massa e por dúzia de ovos, além da gravidade específica, também não foram influenciados pelas diferentes relações met + cis:lis da ração $(\mathrm{P}>0,05)$.

Apesar de os tratamentos não terem afetado a taxa de postura, ocorreu melhora relativa de 1,87; 1,90 e 0,76\% na produção de ovos das aves alimentadas com a relação 0,76 met+cis:lis em comparação à produção das aves nas relações 0,$83 ; 0,85$ e 0,86 , respectivamente.

A massa de ovos foi afetada pelas relações met + cis:lis $(\mathrm{P}<0,05)$, apresentando maior valor para a relação 0,76 $(0,70 \%$ de met + cis e $0,92 \%$ de lis total $)$ em comparação à de

Tabela 4 - Preços dos ingredientes da ração

Table 4 - Prices of diet ingredients

\begin{tabular}{|c|c|}
\hline $\begin{array}{l}\text { Ingrediente } \\
\text { Ingredient }\end{array}$ & $\begin{array}{c}\text { Preço }(\mathrm{kg} / \mathrm{R} \$) \\
\text { Price }\end{array}$ \\
\hline Milho $^{1}$ (Corn) & 0,40 \\
\hline Farelo de soja ${ }^{1}$ (Soybean meal) & 1,00 \\
\hline Glúten de milho ${ }^{1}$ (Corn gluten) & 1,44 \\
\hline Calcário $^{1}$ (Limestone) & 0,12 \\
\hline Fosfato bicálcico ${ }^{1}$ (Dicalcium phosphate) & 1,15 \\
\hline Amido $^{1}$ (Starch) & 4,80 \\
\hline DL-metionina $(99 \%)^{2}$ (DL-methionine) & 8,45 \\
\hline L-lisina $\mathrm{HCl}(78,4 \%)^{2}$ (L-lysine $\mathrm{HCl}$ ) & 11,10 \\
\hline Cloreto de colina $(70 \%)^{1}$ (Choline chloride) & 4,00 \\
\hline Óleo vegetal ${ }^{1}$ (Vegetable oil) & 2,50 \\
\hline Sal comum $^{1}$ (Salt) & 0,20 \\
\hline Premix mineral ${ }^{1}$ (Mineral mix) & 3,20 \\
\hline Premix vitamínico ${ }^{1}$ (Vitamin mix) & 3,00 \\
\hline Areia lavada (Sand washed) & 0,00 \\
\hline
\end{tabular}

$0,83(0,71 \%$ de met+cis e $0,85 \%$ de lis $)$, mas não houve diferença para as relações 0,85 e 0,86 . O maior teor de lisina da dieta foi, provavelmente a causa do melhor resultado de massa de ovos das aves alimentadas com a ração com relação 0,76 , em comparação àquelas recebendo a ração com relação 0,83 de met+cis:lis. Esse resultado corrobora a afirmação de Liu et al. (2004) de que o nível de suplementação de lisina em rações à base de milho e farelo deve ser superior aos níveis normalmente recomendados. Desta forma, o resultado obtido para massa de ovos corrobora a hipótese de que existe uma relação ideal de met+cis:lis que afeta positivamente esta variável por atender melhor às exigências das aves.

A qualidade da casca dos ovos, medida pela gravidade específica, não diferiu entre as diferentes relações met + cis:lis $(\mathrm{P}>0,05)$, justificando que, de modo geral, as especificações de lisina e dos aminoácidos sulfurosos não afetaram a qualidade da casca dos ovos.

A relação 0,76 de met + cis:lis total estimada a partir dos dados publicados por Jordão Filho et al. (2006ab) representa, em termos de aminoácidos totais, uma estimativa de $0,70 \%$ de met + cis e $0,92 \%$ de lis, estando abaixo daquela estimada por Barbosa et al. (1999ab), ao avaliarem o desempenho de poedeiras semipesadas durante o segundo ciclo de postura de $0,81(0,65 \%$ de met + cis e $0,80 \%$ de lis $)$.

Conforme demonstrado na Tabela 6, o peso dos ovos não foi afetado pelas relações met+cis:lis nem pelo armazenamento $(\mathrm{P}>0,05)$. Os pesos e as porcentagens de clara e de gema também não foram influenciados pelas relações dos aminoácidos $(\mathrm{P}>0,05)$, porém foram alterados pelo período de armazenamento $(\mathrm{P}<0,05)$. Com o armazenamento, ocorre redução do peso e da porcentagem de clara, concomitante ao aumento do peso e da porcentagem de gema $(\mathrm{P}<0,05)$. Este resultado pode estar associado à perda de água, resultante das reações físicas e químicas da clara para a gema (Stadelman \& Cotterill, 1995). Segundo Barbosa et al. (2004), esta migração de água da clara para a gema está relacionada a grandes moléculas de proteína que penetram na gema por osmose. Esses autores observaram aumento linear da porcentagem de gema de 0 a 35 dias de estocagem dos ovos. Scott \& Silversides (2000), no entanto, após a estocagem de ovos por dez dias a $20^{\circ} \mathrm{C}$, também observaram diminuição da quantidade de albúmem sem alteração correpondente de gema. A discrepância dos resultados de gema entre este estudo e o de Scott \& Silversides (2000) ocorreu, provavelmente, em função do menor tempo (dez dias) e da maior temperatura $\left(20^{\circ} \mathrm{C}\right)$ de estocagem utilizada por esses autores, de modo que a maior temperatura de exposição dos ovos pode ter aumentado a perda de umidade da clara para 
Tabela 5 - Consumo de ração (CR), produção de ovos (PR), peso de ovos (PO), massa de ovos (MO), conversão por massa de ovos (CMO), conversão por dúzias de ovos (CDZ) e gravidade específica (GE), em função das relações de met+cis:lis das rações experimentais

Table 5 - Feed intake (FI), egg production (EP), egg weight (EW), egg mass (EM), egg mass:feed ratio (EMFR), egg dozen:feed ratio (EDFR) and egg shell specific gravity (SG) according to the dietary met + cys:lis ratios

\begin{tabular}{lccccccccc}
\hline $\begin{array}{l}\text { Trat. } \\
\text { Treat. }\end{array}$ & $\mathrm{M}+\mathrm{C}(\%)$ & Lis $(\%)$ & $\mathrm{CR}(\mathrm{g})$ & $\mathrm{PR}(\%)$ & $\mathrm{PO}(\mathrm{g})$ & $\mathrm{MO}(\mathrm{g})$ & $\mathrm{CMO}(\mathrm{kg} / \mathrm{kg})$ & $\mathrm{CDZ}(\mathrm{kg} / \mathrm{dz})$ & $\mathrm{GE}\left(\mathrm{g} / \mathrm{cm}^{3}\right)$ \\
& & & $F I$ & $E P$ & $E W$ & $E M$ & $E M F R$ & $E D F R$ & $S G$ \\
\hline $0,76^{1}$ & 0,70 & 0,92 & 108,26 & 92,73 & 66,09 & $61,28^{\mathrm{a}}$ & 1,767 & 1,400 \\
$0,83^{2}$ & 0,71 & 0,85 & 107,46 & 91,00 & 64,86 & $59,02^{\mathrm{b}}$ & 1,821 & 1,430 \\
$0,85^{3}$ & 0,64 & 0,75 & 108,50 & 90,97 & 65,59 & $59,67^{\mathrm{ab}}$ & 1,774 & 1,431 \\
$0,86^{4}$ & 0,70 & 0,81 & 109,31 & 92,02 & 65,51 & $60,28^{\mathrm{ab}}$ & 1,810 & 1,086 \\
CV (\%) & & & 1,851 & 2,474 & 2,188 & 2,516 & 2,750 & 2,902 \\
Efeito & & & $\mathrm{ns}$ & $\mathrm{ns}$ & $\mathrm{ns}$ & $*$ & $\mathrm{~ns}$ & $\mathrm{~ns}$ & 0,115 \\
\hline
\end{tabular}

1 Segundo Jordão Filho et al. (2006ab) (According to Jordão Filho et al., 2006ab data).

2 Conforme Manual da linhagem Hisex Brown (1999) (Recommended by Hisex Brown strain handbook, 1999).

3 De acordo com o NRC (1994) (According to NRC, 1994 tables).

4 Valores constantes em Rostagno et al. (2000) (Values recommended by Rostagno et al., 2000).

ns não-significativo (not significant).

o ambiente. Conforme Stadelman \& Cotterill (1995), a temperatura ambiente é um dos principais fatores de redução da umidade dos ovos com aumento do tempo de armazenamento.

As relações aminoacídicas e o período de estocagem não afetaram o $\mathrm{pH}$ da clara $(\mathrm{P}>0,05)$. Apesar disso, ocorreu moderado aumento no $\mathrm{pH}$ deste componente com o armazenamento dos ovos durante 28 dias, causado, provavelmente, pela perda do dióxido de carbono através dos poros da casca. Segundo Stadelman \& Cotterill, (1995), o pH do albúmem é dependente do equilíbrio entre íons carbonatos, íons bicarbonados, proteína e dióxido de carbono.

Ao contrário da clara, o $\mathrm{pH}$ da gema cresceu significativamente após o período de armazenamento $(\mathrm{P}<0,05)$, o que parece corroborar a afirmação de Shang et al. (2004) de que, durante a armazenagem sob refrigeração (28 dias), íons alcalinos, como sódio, potássio e magnésio migram do albúmem para a gema, trocados pelos íons hidrogênio, provocando o aumento do $\mathrm{pH}$ da gema. O aumento do pH da gema causa a dissociação do complexo ovomucinalisozima, melhorando a ação da lisozima contra a proliferação de bactérias no interior da gema (Ruiz, 1992), e, portanto, $\mathrm{pH}$ elevado dificulta a resistência das bactérias. Segundo Ruiz (1992), isto é possível porque a lisozima é uma enzima capaz de cindir a camada mucopeptídica da parede celular de bactérias, tornando-as sensíveis ao choque osmótico.

Os valores de $\mathrm{pH}$ da gema e da clara estão de acordo com aqueles descritos por Shang et al. (2004), de 6,21 a 7,39 para o $\mathrm{pH}$ da gema e de 7,72 a 9,28 para o $\mathrm{pH}$ da clara.

Os valores absolutos e relativos da casca dos ovos (Tabela 6) não foram afetados pelas relações met+cis:lis $(\mathrm{P}>0,05)$ nem pelo período de armazenamento, indicando que a relação dos aminoácidos estudados e o tempo de armazenamento não alteram a qualidade externa do ovo. A gravidade específica não foi afetada pelas relações met + cis:lis $(\mathrm{P}>0,05)$, mas verificou-se efeito significativo dos períodos de estocagem sobre esta variável, que piorou com o armazenamento dos ovos. Provavelmente, isso ocorreu mais pelo aumento da câmara de ar com a perda de umidade da clara para o meio externo que pela diminuição na resistência da casca. Resultado semelhante foi citado por Carvalho et al. (2003), que observaram efeito negativo do período de armazenamento, de 1, 5, 9, 13 e 16 dias sobre a gravidade específica, que diminuiu de 1,077 para 1,074; 1,$067 ; 1,066$ e 1,065, respectivamente. Outros autores também observaram que a temperatura e o tempo de armazenamento afetam a qualidade interna dos ovos, enquanto a qualidade externa não é alterada (Scott \& Silversides, 2000).

A relação gema: clara foi alterada após o período de estocagem $(\mathrm{P}<0,05)$, mas não foram influenciadas pelas relações dos aminoácidos estudadas $(\mathrm{P}>0,05)$, o que sugere que o conteúdo de aminoácidos da dieta, ao contrário do tempo de armazenamento dos ovos, não afeta estas características, resultando em aumento da proporção de gema e redução do conteúdo de clara.

Os potenciais de óxido-redução (Eh) da clara e da gema não foram influenciados $(\mathrm{P}>0,05)$ pelas relações met + cis:lis (Tabela 6). Entretanto, o Eh da gema foi reduzido pelo período de estocagem $(\mathrm{P}<0,05)$, o que indica possível oxidação da fração lipídica da gema durante a estocagem. Segundo Hayes (1993), a alteração da qualidade dos ovos deve-se fundamentalmente às bactérias gram-positivas. $\mathrm{O}$ armazenamento em condições controladas de temperatura e de umidade retarda a velocidade de deterioração da qualidade da gema dos ovos, mas alterações importantes devem ocorrer na gema, como conseqüência da alta concentração de ácidos graxos insaturados desta fração do ovo. 


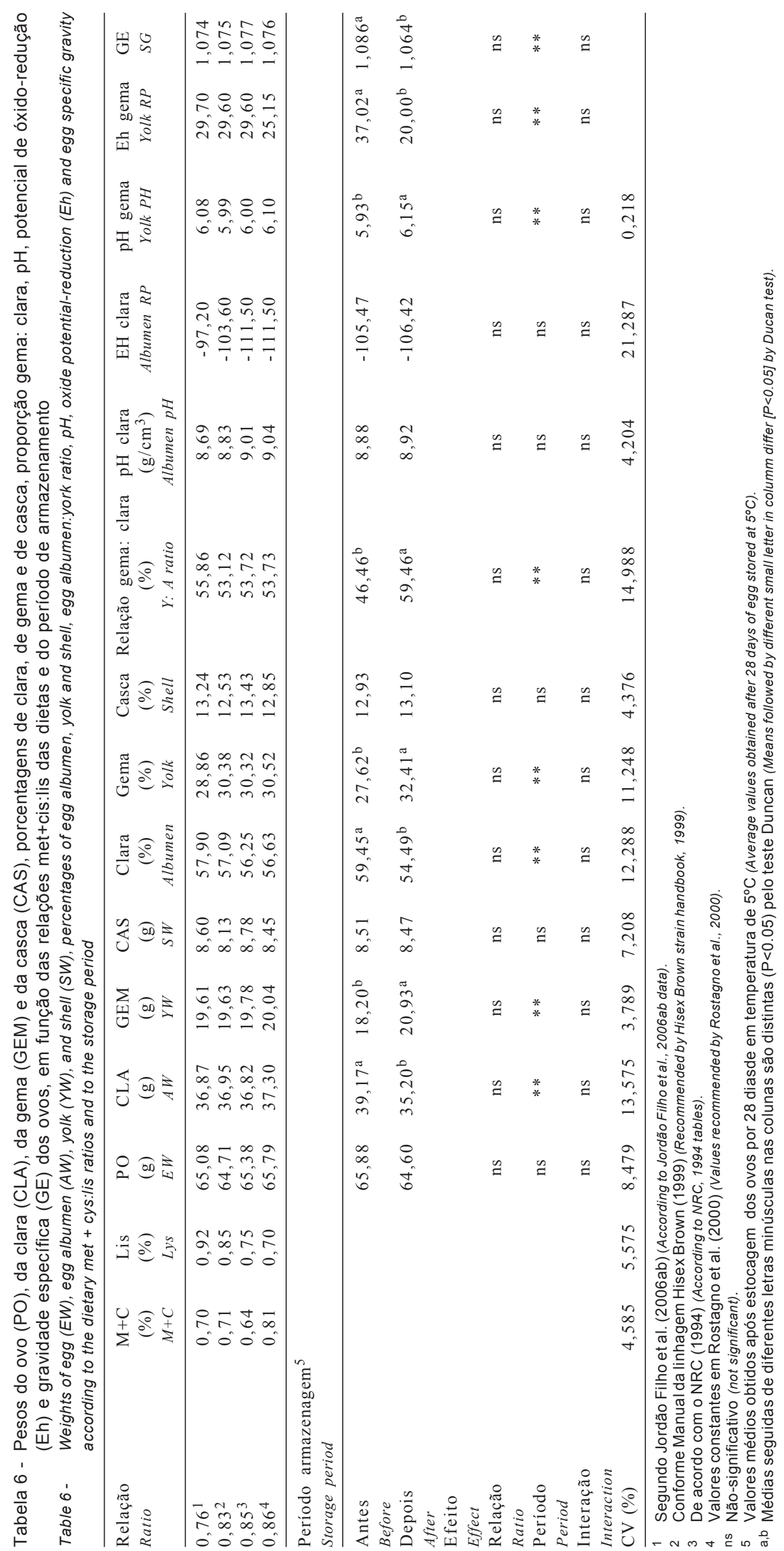


Tabela 7 - Renda bruta média $\left(\mathrm{RBM}^{1}\right)$, custo médio de arraçoamento (CMA), margem bruta média (MBM) e rentabilidade média do ovo (RMO) em função das relações de met+cis:lis das rações experimentais

Table 7 - Average gross income (AGI), average feeding cost (AFC), average gross margin ( $A G M)$, and average egg profitability (AEP) according to the dietary met + cys:lis ratios

\begin{tabular}{|c|c|c|c|c|c|c|c|c|c|}
\hline $\begin{array}{l}\text { Tratamento } \\
\text { Treatement }\end{array}$ & $\begin{array}{c}\mathrm{M}+\mathrm{C}(\%) \\
M+C\end{array}$ & $\begin{array}{l}\text { Lis }(\%) \\
\text { Lysine }\end{array}$ & $\begin{array}{c}\text { Preço }(\mathrm{R} \$ / \mathrm{kg}) \\
\text { Price }\end{array}$ & $\begin{array}{c}\mathrm{CRT}(\mathrm{g} / \mathrm{a} / \mathrm{d}) \\
\text { FIT }\end{array}$ & $\begin{array}{c}\text { Caixa/ovos (30) } \\
\text { Box/egg }\end{array}$ & $\begin{array}{c}\mathrm{RBM} \\
A G I\end{array}$ & $\begin{array}{l}\text { CMA } \\
A F C\end{array}$ & $\begin{array}{l}\mathrm{MBM} \\
A G M\end{array}$ & $\begin{array}{l}\mathrm{RMO} \\
A E P\end{array}$ \\
\hline $0,76^{1}$ & 0,71 & 0,90 & 0,6350 & 52,701 & 15,000 & 55,500 & 33,465 & 22,035 & 65,845 \\
\hline $0,83^{2}$ & 0,71 & 0,85 & 0,6336 & 52,064 & 14,613 & 54,068 & 32,988 & 21,080 & 63,092 \\
\hline $0,85^{3}$ & 0,64 & 0,75 & 0,6186 & 52,399 & 14,553 & 53,846 & 32,414 & 21,432 & 66,119 \\
\hline $0,86^{4}$ & 0,70 & 0,81 & 0,6268 & 52,543 & 14,453 & 53,476 & 32,934 & 20,542 & 62,373 \\
\hline $\mathrm{CV}(\%)$ & & & & & & 6,344 & 2,525 & 13,477 & 12,030 \\
\hline Efeito & & & & & & ns & ns & $\mathrm{ns}$ & $\mathrm{ns}$ \\
\hline
\end{tabular}

Effect

Obtido com preço de venda da caixa de 30 ovo por $\mathrm{R} \$ 3,70$.

2 Segundo Jordão Filho et al. (2006ab) (According to Jordão Filho et al., 2006ab data).

3 Conforme Manual da linhagem Hisex Brown (1999) (Recommended by Hisex Brown strain handbook, 1999).

4 De acordo com o NRC (1994) (According to NRC, 1994 tables).

5 Valores constantes em Rostagno et al. (2000) (Values recommended by Rostagno et al., 2000).

ns Não-significativo (not significant).

Ovos estocados por longos períodos têm sua qualidade interna alterada (Brugalli et al., 1998; Scott \& Silversides, 2000; Faria et al., 2003), perdendo, possivelmente, a qualidade para uso na indústria alimentícia.

$\mathrm{Na}$ Tabela 7, encontram-se os índices econômicos obtidos com as diferentes recomendações de relação met + cis:lis para poedeiras semipesadas.

Considerando os resultados da análise econômica, observa-se que as diferentes relações met + cis:lis não afetaram nenhuma das características avaliadas $(\mathrm{P}>0,05)$. Embora não tenha sido estatisticamente comprovado, o custo médio de arraçoamento foi maior para a relação 0,76 , em virtude da maior quantidade de L-lisina $\mathrm{HCl}$ suplementar, que justifica a afirmativa de Yadalan et al. (2000) de que a suplementação de lis e met+cis, quando em níveis elevados, onera o custo da ração. No entanto, considerando o preço estimado das rações e o preço do ovo em $\mathrm{R} \$ 3,70 / 30$ unidades (Tabela 7), observa-se que não houve melhora significativa do retorno econômico, em porcentagem, sobre o custo de arraçoamento, indicando que quaisquer das dietas poderiam ser utilizadas.

A relação met+cis:lis de 0,76 continha maior teor de L-lisina $\mathrm{HCl}$ suplementar, apresentando preço por quilograma de ração de $\mathrm{R} \$ 0,6350$, enquanto as relações do Interaves (1999), NRC (1994) e Rostagno et al. (2000) apresentaram preços de $\mathrm{R} \$ 0,6336 ; 0,6186$ e $0 ; 6268$ (Tabela 1), respectivamente. Independentemente da influência no preço final da ração, as exigências das aves em lisina $(0,92 \%)$ e metionina + cistina $(0,70 \%)$, para melhor retorno econômico, parecem ser possíveis a partir de dieta contendo relação 0,76 met+cis: lis, recomendada por Jordão Filho et al. (2006ab). $\mathrm{O}$ teor de lisina de $0,92 \%$ é superior às recomendações do NRC (1994), 0,71\%, da Interaves (1999), 0,85\%, e de Rostagno et al. (2000), 0,81\%. Este resultado difere daquele descrito por Yadalan et al. (2000), que criticaram os níveis de met + cis:lis sugeridos pelo NRC (1994) por onerar o custo da ração em U\$1,0/t. Essa divergência ocorre porque esses autores não consideraram o retorno econômico com a produção e venda de ovos produzidos com níveis mais altos de aminoácidos da dieta.

Portanto, a escolha de dieta para otimizar o retorno financeiro não depende apenas do custo final da ração, mas, sobretudo, do desempenho produtivo e do custo dos ovos no mercado.

\section{Conclusões}

Rações com relação 0,76 ou $0,70 \%$ de metionina+cistina total $(0,64 \%$ de metionina+cistina digestível) e $0,92 \%$ de lisina total $(0,84 \%$ de lisina digestível $)$ otimizam os desempenhos produtivo e econômico de poedeiras semipesadas.

O armazenamento durante 28 dias em condições de geladeira diminui a qualidade interna dos ovos.

\section{Agradecimento}

Ao laboratorista do CFT/UFPB, Jerônimo Galdino dos Santos, pela contribuição nas análises químicas.

\section{Literatura Citada}

BARBOSA, N.A.A.; FREITAS, E.R.; SAKOMURA, N.K. et al. Efeito da temperatura e do tempo de armazenagem na qualidade interna de ovo de poedeiras comerciais. Revista Brasileira de Ciência Avícola, suplemento 6, 2004, p.60.

BARBOSA, B.A.C.; SOARES, P.R.; ROSTAGNO, H.S. et al. Exigência nutricional de metionina +cistina para galinhas poedeiras de ovos brancos e marrons, no segundo ciclo de produção. 1. Características produtivas. Revista Brasileira de Zootecnia, v.28, n.3, p.526-533, 1999a. 
BARBOSA, B.A.C.; SOARES, P.R.; ROSTAGNO, H.S. et al. Exigência nutricional de lisina para galinhas poedeiras de ovos brancos e ovos marrons, no segundo ciclo de produção. 2. Características produtivas. Revista Brasileira de Zootecnia, v.28, n.3, p.534-541, 1999b.

BRUGALLI, I.; RUTZ, F.; ZONTA, E.P. et al. efeito dos níveis de óleo e proteína da dieta sobre a qualidade interna de ovos, em diferentes condições e tempo de armazenamento. Revista Brasileira de Agrociência, v.4, n.3, p.187-190, 1998.

CARVALHO, F.B.; STRINGHINI, J.H.; LEANDRO, N.S.M. et al. Influencia das linhagens e idades de poedeiras comerciais na qualidade interna e da casca para ovos armazenados sob diferentes temperaturas. Revista Brasileira de Ciência Avícola, Suplemento 5, 2003, p.101.

EISEN, E.J.; BOHREN, B.B.; McKEAN, H.E. The Haugh unit as a measure of egg albumen quality. Poultry Science, v.41, n.5, p.1461-1468, 1962 .

FARIA, D.E.; FARIA FILHO, D.E.; RIZZO, M.F. Qualidade do ovo para industrialização. In: CONFERÊNCIA APINCO DE CIÊNCIAS E TECNOLÓGIA AVÍCOLA, 2003, Campinas. Anais de Palestra... Campinas: Fundação Apinco de Ciência e Tecnologia Avícolas, 2003. p.325-346.

GUIDONI, A.L. Alternativas para comparar tratamentos envolvendo o desempenho nutricional. Piracicaba: Escola Superior de Agricultura "Luiz de Queiroz", 1994. 105p. Tese (Doutorado em Estatística e Experimentação Agronômica) - Escola Superior de Agricultura "Luiz de Queiroz", 1994.

HAYES, P.R. Microbiologia e higiene de los alimentos: el huevo de gallina y su alteración. Zaragoza: Acribia, 1993. p.103106.

INTERAVES. Manual de produção Hisex Brown. 1.ed. Cascavel: 1999. 43p.

JORDÃO FILHO, J.; SILVA, J.H.V.; SILVA, E.L. et al. Exigência de lisina para poedeiras semipesadas durante o pico de postura. Revista Brasileira de Zootecnia, v.35, n.4, p.1726-1732, 2006 a.

JORDÃO FILHO, J.; SILVA, J.H.V.; SILVA, E.L. et al. Exigência nutricionais em metionina + cistina para poedeiras semipesadas do início de produção até o pico de postura. Revista Brasileira de Zootecnia, v.35, n.3, p.1063-1069, 2006b (supl.).

KIDWELL, M.G.; NORDSKOG, A.W.; FORSYTHE, R.H. On the problem of correcting albumen quality measures for egg weight. Poultry Science, v.43, n.1, p.42-49, 1964.

LIU, Z.; WU, G.; BRYANT, M.M. et al. Influence of added synthetic lysine for first phase second cycle commercial leghorns with the methionine + cystine/Lisine ratio mainained at 0.75 . International Journal of Poultry Science, v.3, n.3, p.220$227,2004$.

NATIONAL RESEARCH COUNCIL - NRC. Nutrient requeriments of poultry. 9.rev.ed. Washington, D.C.: National Academy Press, 1994. $155 \mathrm{p}$.

NUNES, I.J. Nutrição animal básica. 2.ed. Belo Horizonte: FEP - MVZ, 1998. 388p.

PROCHASKA, J.F.; CAREY, J.B.; SHAFER, D.J. The effect of L-lysine intake on egg component yield and composition in laying hens. Poultry Science, v.75, n.10, p.1268-1277, 1996.

ROSTAGnO, H.S.; ALBINO, L.F.T.; DONZELE, J.L. et al. Tabelas brasileiras para suínos e aves: composição de alimentos e exigências nutricionais. Viçosa, MG: Universidade Federal de Viçosa, 2000. 141p.

RUIZ, R.L. Microbiologia zootécnica. São Paulo: Roca, 1992. $314 \mathrm{p}$.

SCOTT, T.A.; SILVERSIDES, F.G. The effect of storage strain of hen on egg quality. Poultry Science, v.79, n.12, p.17251729,2000 .
SHAFER, D.J.; CAREY, J.B.; PROCHASKA, J.F. Effect of dietary methionine intake on egg component yield and composition. Poultry Science, v.75, n.9, p.1080-1085, 1996.

SHAFER, D.J.; CAREY, J.B.; PROCHASKA, J.F. et al. Dietary methionine intake effects on egg component yield, composition, functionality, and texture profile analysis. Poultry Science, v.77, n.7, p.1056-1062, 1998.

SHANG, X.G.; WANG, F.L.; LI, D.F. et al. Effects of dietary conjugated linoleic acid on the productivity of laying hens and egg quality during refrigerated storage. Poultry Science, v.83, n. 10, p.1688-1695. 2004

SILVA, J.H.V.; ALBINO, L.F.T.; ROSTAGNO, H.S. et al. Exigência de lisina para aves de reposição de 0 a 6 semanas de idade. Revista Brasileira de Zootecnia, v.29, n.6, p.1777-1785, 2000a.

SILVA, J.H.V.; ALBINO, L.F.T.; ROSTAGNO, H.S. et al. Exigência de lisina para aves de reposição de 7 a 12 semanas de idade. Revista Brasileira de Zootecnia, v.29, n.6, p.1795-1802, 2000 b.

SILVA, J.H.V.; ALBINO, L.F.T.; ROSTAGNO, H.S. et al. Exigência de lisina para aves de reposição de 13 a 20 semanas de idade. Revista Brasileira de Zootecnia, v.29, n.6, p.1795-1802, $2000 \mathrm{c}$

SILVA, J.H.V.; LIMA, M.R.; ARAÚJO, J.A. et al. Relacionamento lisina e arginina digestível para poedeiras durante o pico de postura. In: CONGRESSO NORDESTINO DE PRODUÇÃO ANIMAL, 3., 2004, Campina Grande. Anais... Campina Grande: Sociedade Nordestina de Produção Animal, 2004a. CD-ROM. Nutrição de não-ruminantes.

SILVA, J.H.V.; JORDÃO FILHO, J.; SILVA, E.L. et al. Exigências de metionina+cistina de poedeiras semipesadas mantendo ou não a relação aminoácidos sulfurosos: lisina. Revista Brasileira de Ciência Avícola, v.4, p.69, 2002 (suplemento).

SILVA, J.H.V.; SILVA, A.C.S.; LIMA, M.R. et al. Níveis de aminoácidos digestíveis na dieta de cria de pintainhas semipesadas. In: CONGRESSO NORDESTINO DE PRODUÇÃO ANIMAL, 3., 2004, Campina Grande. Anais... Campina Grande: Sociedade Nordestina de Produção Animal, 2004b. CD-ROM. Nutrição de não-ruminantes.

SILVA, J.H.V.; JORDÃO FILHO, J.; SILVA, E.L. Por que formular dietas para poedeiras com base no conceito de proteína ideal? Revista Ave World, v.3, n.3, p.50-57, 2005.

SILVERSIDES, F.G.; TWIZEYIMANA, F.; VILLENEUVE, P. Research note: a study relating to the validity of the Haugh unit correction for egg weight in fresh eggs. Poultry Science, v.72, n.4, p.760-764, 1993

SILVERSIDES, F.G.; VILLENEUVE, P. Is the Haugh unit correction for egg weight valid for eggs stored at room temperature? Poultry Science, v.73, n.1, p.50-55,1994

STADELMAN, W.J.; COTTERILL, O.J. Egg science and technology. 4.ed. New York: Food Products Press, 1995. 591p.

UNIVERSIDADE FEDERAL DE VIÇOSA - UFV. SAEG - Sistema para Análise Estatística e Genética. Versão 5.0. Viçosa, MG: Fundação Arthur Bernardes, 1993.

YADALAN, S.; BRYANT, M.; ROLAND, D.A. Optimal M+C/Lisine ratio for molted phase $1 \mathrm{Hy}-$ line 36 hens: Poultry Science, v.79, p.92, 2000 (suppl.). 